\title{
PERTURBATIONS IN A SIGNED GRAPH AND ITS INDEX
}

\author{
ZORAN STANić ${ }^{1}$ \\ Faculty of Mathematics \\ University of Belgrade \\ Studentski trg 16, 11000 Belgrade, Serbia \\ e-mail: zstanic@math.rs
}

\begin{abstract}
In this paper we consider the behaviour of the largest eigenvalue (also called the index) of signed graphs under small perturbations like adding a vertex, adding an edge or changing the sign of an edge. We also give a partial ordering of signed cacti with common underlying graph by their indices and demonstrate a general method for obtaining lower and upper bounds for the index. Finally, we provide our computational results related to the generation of small signed graphs.
\end{abstract}

Keywords: signed graph, switching equivalence, index, computer search.

2010 Mathematics Subject Classification: 05C50, 05C30, 65F15.

\section{REFERENCES}

[1] F. Belardo, E.M. Li Marzi and S.K. Simić, Combinatorial approach for computing the characteristic polynomial of a matrix, Linear Algebra Appl. 433 (2010) 15131523.

doi:10.1016/j.laa.2010.05.010

[2] F. Belardo and P. Petecki, Spectral characterizations of signed lollipop graphs, Linear Algebra Appl. 480 (2015) 144-167.

doi:10.1016/j.laa.2015.04.022

[3] D. Cvetković, M. Doob and H. Sachs, Spectra of Graphs - Theory and Application, 3rd Edition (Johann Ambrosius Barth Verlag, Heidelberg-Leipzig, 1995).

[4] D. Cvetković, P. Rowlinson and S. Simić, An Introduction to the Theory of Graph Spectra (Cambridge University Press, Cambridge, 2010).

\footnotetext{
${ }^{1}$ Research is partially supported by Serbian Ministry of Education, Science and Technological Development, Projects 174012 and 174033.
} 
[5] W.H. Haemars and E. Spence, Enumeration of cospectral graphs, European J. Combin. 25 (2004) 199-211.

doi:10.1016/S0195-6698(03)00100-8

[6] T. Koledin and Z. Stanić, Connected signed graphs of fixed order, size, and number of negative edges with maximal index, Linear Multilinear Algebra 65 (2017) 21872198.

doi:10.1080/03081087.2016.1265480

[7] B.D. McKay and A. Piperno, Practical graph isomorphism, II, J. Symbolic Comput. 60 (2014) 94-112.

doi:10.1016/j.jsc.2013.09.003

[8] G. Pólya, Kombinatorische Anzahlbestimmungen für Gruppen, Graphen und chemische Verbindungen, Acta Math. 68 (1937) 145-254. doi:10.1007/BF02546665

[9] S.K. Simić and Z. Stanić, Polynomial reconstruction of signed graphs, Linear Algebra Appl. 501 (2016) 390-408. doi:10.1016/j.laa.2016.03.036

[10] Z. Stanić, Inequalities for Graph Eigenvalues (Cambridge University Press, Cambridge, 2015).

Received 30 May 2016

Revised 23 February 2017

Accepted 23 February 2017 